\title{
$C l^{\star}$-CONNECTEDNESS AND $C l-C l^{\star}$-CONNECTEDNESS IN
} IDEAL TOPOLOGICAL SPACES

\author{
B. K. TYAGI, MANOJ BHARDWAJ, AND SUMIT SINGH
}

\begin{abstract}
In this paper, new types of connectedness of ideal topological space $X$ and their relationships with other existing connectednesses are discussed.
\end{abstract}

\section{INTRODUCTION}

The concept of an ideal topological space was introduced by Kuratowski [6] and Vaidyanathswamy [10]. An ideal $I$ on a set $X$ is a nonempty collection of subsets of $X$ which satisfies $(i) A \in I$ and $B \subset A$ implies $B \in I$ and (ii) $A \in I$ and $B \in I$ implies $A \cup B \in I$. For a subset $A$ of $X$, the local function of $A$ is defined as follows [3, 4]: $A^{\star}=\{x \in X: U \cap A \notin I$ for every $U \in \tau(x)\}$, where $\tau(x)$ is the collection of all nonempty open sets containing $x$. The study of $\star$-topology is done by Jankovic and Hamlett $[4,5]$, Modak and Bandyopadhyay $[7,8]$ in detail. Its one of the powerful base is $\beta(I, \tau)=\{V \backslash A: V \in \tau, A \in I\}$ [4]. It is also denoted as $\tau^{\star}(I)$ $[4,5]$ and its closure operator is defined as $C l^{\star}(A)=A \cup A^{\star}$. It is known that $\tau \subset \tau^{\star}(I)$. The elements of $\tau^{\star}(I)$ are called $\star$-open sets and their complements are called $\star$-closed to differentiate them from open and closed sets in $X$. If an ideal satisfies $I \cap \tau=\{\emptyset\}$, then the ideal is termed as codense ideal by Dontchev, Ganster and Rose [1]. An ideal $I$ is compatible [5] with the topology $\tau$ written as $I \sim \tau$ [5] if for any $A \subset X$ there is an open cover $\Omega_{x}$ of $A$ such that for $x \in A$, there is a $U_{x} \in \Omega_{x}$ with $U_{x} \cap A \in I$, then $A \in I$. The study of connectedness as $\star_{s}$-connectedness and $\star$-connectedness in an ideal topological space was introduced by Ekici and Noiri in [2]. The authors Sathiyasundari and Renukadevi [9] studied it further in detail. The authors Modak and Noiri [11] introduced some different types of connectedness as $\star_{\star}$-connectedness, $\star-C l$-connectedness and $\star-C l^{\star}$-connectedness with the help of the ideal topological spaces. In this paper we introduced some different types of connectedness in ideal topological spaces and interrelated with earlier existing connectedness of Ideal topological spaces. We studied the notions of $C l^{\star}$-connected sets and

2010 Mathematics Subject Classification. 54A05, 54D05.

Key words and phrases. $\star_{\star}$-connected, $\star$-connected, $C l^{\star}$-connected, $\star-C l^{\star}$ connected, $\mathrm{Cl}-\mathrm{Cl}^{\star}$-connected.

The second (corresponding) and third authors acknowledgement the fellowship grant of University Grant Commission, India. 
$C l-C l^{\star}$-connected sets in ideal topological spaces.

This paper is organised as follows. In Section 2, the relationships between the new types of connectedness and existing connectedness in literature, are developed. In Section 3, the basic properties of $C l^{\star}$-connected spaces and $C l-C l^{\star}$-connected spaces are discussed. It covers the concept of $C l^{\star}$ component and $\mathrm{Cl}-\mathrm{Cl} l^{\star}$-component.

\section{Preliminaries}

Let $(X, \tau)$ or $X$ be a topological space or a space. We will denote by $C l(A)$ and $\operatorname{Int}(A)$ the closure of $A$ and the interior of $A$, for a subset $A$ of $X$, respectively. Through out the paper, by subset $A$ of an ideal topological space $(X, \tau, I)$, always refers to a subset of $X$.

Definition 1. Nonempty subsets $A, B$ of an ideal space $(X, \tau, I)$ are called

(1) $\star_{\star}$-separated ([11]) if $A^{\star} \cap B=A \cap B^{\star}=A \cap B=\emptyset$.

(2) $\star$-separated ([2]) if $C l^{\star}(A) \cap B=A \cap C l(B)=\emptyset$.

(3) separated if $C l(A) \cap B=A \cap C l(B)=\emptyset$.

Definition 2. Nonempty subsets $A, B$ of an ideal space $(X, \tau, I)$ are called

(1) $\star-C l$-separated ([11]) if $A^{\star} \cap C l(B)=C l(A) \cap B^{\star}=A \cap B=\emptyset$.

(2) $\star-C l^{\star}$-separated ([11]) if $\left.A^{\star} \cap C l^{\star}(B)=C l^{\star}(A) \cap B^{\star}=A \cap B=\emptyset\right)$.

Definition 3. Nonempty subsets $A, B$ of an ideal space $(X, \tau, I)$ are called

(1) $C l^{\star}$-separated if $A \cap C l^{\star}(B)=C l^{\star}(A) \cap B=A \cap B=\emptyset$.

(2) $C l-C l^{\star}$-separated if $\left.C l(A) \cap C l^{\star}(B)=C l^{\star}(A) \cap C l(B)=A \cap B=\emptyset\right)$.

Proposition 1. ([4]) Let $(X, \tau)$ be a space with $I_{1}$ and $I_{2}$ being ideals on $X$, and let $A$ and $B$ be two subsets on $X$. Then

(a) $A \subseteq B \Rightarrow A^{\star} \subseteq B^{\star}$;

(b) $I_{1} \subseteq I_{2} \Rightarrow A^{\star}\left(I_{2}\right) \subseteq B^{\star}\left(I_{1}\right)$;

(c) $A^{\star}=C l\left(A^{\star}\right) \subseteq C l(A)$;

(d) $\left(A^{\star}\right)^{\star} \subseteq A^{\star}$;

(e) $(A \cup B)^{\star}=A^{\star} \cup B^{\star}$;

(f) $A^{\star} \backslash B^{\star}=(A \backslash B)^{\star} \backslash B^{\star} \subseteq(A \backslash B)^{\star}$;

(g) for every $D \in I,(A \cup D)^{\star}=A^{\star}=(A \backslash D)^{\star}$.

Proposition 2. ([11]) Let $(X, \tau, I)$ be an ideal topological space and $A, B \subset$ $X$. Then $A$ and $B$ are $\star_{\star^{-}}$separated if and only if $A$ and $B$ are separated in $\left(X, \tau^{\star}(I)\right)$.

Proposition 3. ([11]) For nonempty subsets of an ideal space $(X, \tau, I)$, the followings hold:

(a) Every $\star-C l^{\star}$-separated set is $\star_{\star}$-separated.

(b) Every $\star-C l$-separated set is $\star-C l^{\star}$-separated.

Theorem 1. For nonempty subsets of an ideal space $(X, \tau, I)$, the following statements hold:

(a) $C l-C l^{\star}$-separated sets are separated.

(b) $C l-C l^{\star}$-separated sets are $\star-C l$-separated. 
(c) $\star-C l$-separated sets are $\star-C l^{\star}$-separated.

(d) $\star-C l^{\star}$-separated sets are $\star_{\star}$-separated.

(e) separated sets are $\star$-separated.

(f) $\star$-separated sets are $C l^{\star}$-separated.

(g) $C l^{\star}$-separated sets are $\star_{\star}$-separated.

(h) $\star_{\star}$-separated sets are $C l^{\star}$-separated

(i) $C l^{\star}$-separated sets are separated in $\left(X, \tau^{\star}(I)\right)$.

(j) separated sets in $\left(X, \tau^{\star}(I)\right)$ are $C l^{\star}$-separated.

Proof. Follows directly from the definitions.

Thus, the following diagram is obtained:

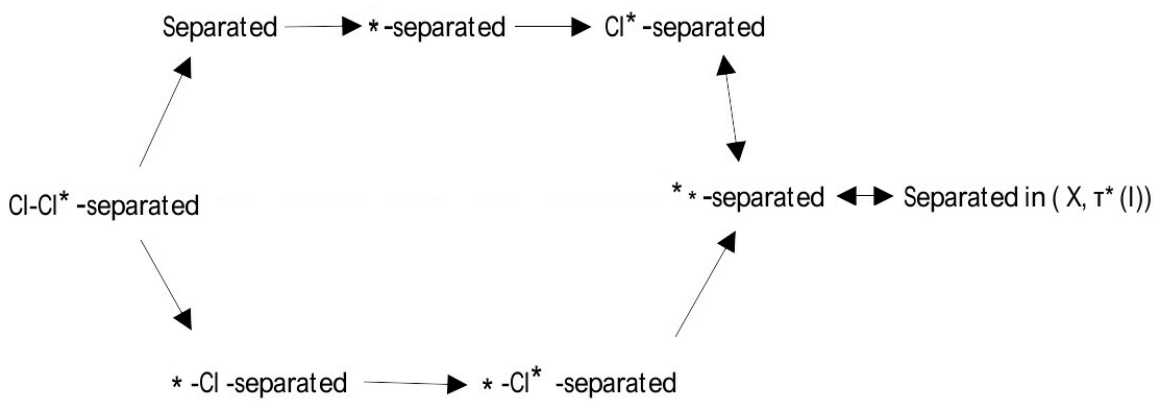

$A \rightarrow B$ means $A$ implies $B$. For converse of Theorem 1 we shall give followings examples.

Example 1. Let $\mathbb{R}$ be the set of real numbers with usual topology on it and $I=\{\emptyset,((b-c) / 2,(b+c) / 2), S$ : where $S$ is subset of $((b-c) / 2,(b+c) / 2)$ and $b, c \in \mathbb{R}$ with $b>c>0\}$ be an ideal on $\mathbb{R}$. Then $A=[b, c)$ and $B=(a, b)($ where $a<(b-c) / 2)$ are $C l^{\star}$-separated in $\mathbb{R}$ but not $\star$-separated in $\mathbb{R}$.

Example 2. In example 1, $A=(a, b)$ and $B=[b, c)$ are $\star-C l$-separated in $\mathbb{R}$ but not $C l-C l^{\star}$-separated in $\mathbb{R}$.

Example 3. Let $\mathbb{R}$ be the set of real numbers with usual topology on it and $I=\{\emptyset\} \cup\{\{b\} \mid b \in \mathbb{R}\}$ be an ideal on $\mathbb{R}$. Then $A=(a, b)$ and $B=(b, c)$ are separated in $\mathbb{R}$ but not $C l-C l^{\star}$-separated in $\mathbb{R}$.

Definition 4. A subset $A$ of an ideal space $(X, \tau, I)$ is called

(a) $\star_{\star}$-connected $([11])\left(\right.$ resp. $\star_{s}$-connected $\left.([2])\right)$ if $A$ is not the union of two $\star_{\star}$-separated (resp. $\star$-separated) sets in $(X, \tau, I)$.

(b) $\star$-connected ([12]) if A cannot be written as the union of a nonempty open set and a nonempty $\star$-open set.

Proposition 4. ([11]) Let $(X, \tau, I)$ be an ideal topological space. Then the space $X$ is $\star_{\star}$ - connected if and only if $\left(X, \tau^{\star}(I)\right)$ is connected.

Definition 5. ([11]) A subset $A$ of an ideal space $(X, \tau, I)$ is called $\star-C l$ connected (resp. $\star-C l^{\star}$-connected) if $A$ is not the union of two $\star-C l$ separated (resp. $\star-C l^{\star}$-separated) sets in $(X, \tau, I)$. 
Definition 6. A subset $A$ of an ideal space $(X, \tau, I)$ is called

(a) $C l^{\star}$-connected if $A$ is not the union of two $C l^{\star}$-separated sets in $(X, \tau, I)$.

(b) $C l-C l^{\star}$-connected if $A$ is not the union of two $C l-C l^{\star}$-separated sets in $(X, \tau, I)$.

Proposition 5. ([11]) For nonempty subsets of an ideal space $(X, \tau, I)$, the followings hold:

(a) Every $\star_{\star}$-connected sets are $\star-C l^{\star}$-connected.

(b) Every $\star-C l^{\star}$-connected sets are $\star-C l$-connected.

Theorem 2. For nonempty subsets of an ideal space $(X, \tau, I)$, the following statements hold:

(a) Every $\star-C l$-connected set is $C l-C l^{\star}$-connected.

(b) Every $\star-C l^{\star}$-connected set is $\star-C l$-connected.

(c) Every $\star_{\star}$-connected set is $\star-C l^{\star}$-connected.

(d) Every connected set is $\mathrm{Cl}-\mathrm{Cl} l^{\star}$-connected.

(e) Every $\star_{s}$-connected set is connected.

(f) Every $C l^{\star}$-connected set is $\star_{s}$-connected.

(g) Every $\star_{\star}$-connected set is $\mathrm{Cl}^{\star}$-connected.

(h) Every $C l^{\star}$-connected set is $\star_{\star}$-connected.

(i) Every connected set in $\left(X, \tau^{\star}(I)\right)$ is $C l^{\star}$-connected and conversely.

(j) Every $C l^{\star}$-connected set is connected set in $\left(X, \tau^{\star}(I)\right)$.

Proof. Follows directly from the definitions, and Theorem 1.

Hence by Theorem 2, we obtain the following diagram:

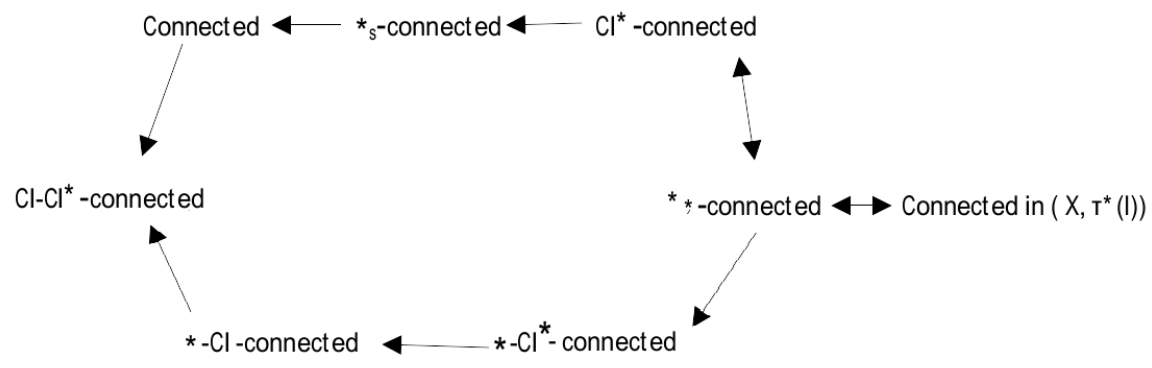

\section{3. $C l^{\star}$-Connected Spaces and $C l-C l^{\star}$-Connected Spaces}

Theorem 3. Let $(X, \tau, I)$ be an ideal space. If $A$ is a $C l^{\star}$-connected subset of $X$ and $H, G$ are $C l^{\star}$-separated sets of $X$ with $A \subset H \cup G$, then either $A \subset H$ or $A \subset G$.

Proof. Let $A \subset H \cup G$. Since $A=(A \cap H) \cup(A \cap G)$, and $(A \cap G) \cap C l^{\star}(A \cap$ $H) \subset G \cap C l^{\star}(H)=\emptyset$. Similarly, $(A \cap H) \cap C l^{\star}(A \cap G)=\emptyset$. Moreover $(A \cap H) \cap(A \cap G) \subset H \cap G=\emptyset$. Suppose that $A \cap H$ and $A \cap G$ are 
nonempty. Then $\mathrm{A}$ is not a $C l^{\star}$-connected, a contradiction. Thus, either $A \cap H=\emptyset$ or $A \cap G=\emptyset$.

Theorem 4. Let $(X, \tau, I)$ be an ideal space. If $A$ is a $C l-C l^{\star}$-connected subset of $X$ and $H, G$ are $C l-C l^{\star}$-separated sets of $X$ with $A \subset H \cup G$, then either $A \subset H$ or $A \subset G$.

Proof. Let $A \subset H \cup G$. Since $A=(A \cap H) \cup(A \cap G)$, and $C l(A \cap G) \cap$ $C l^{\star}(A \cap H) \subset C l(G) \cap C l^{\star}(H)=\emptyset$. Similarly, $C l(A \cap H) \cap C l^{\star}(A \cap G)=\emptyset$. Moreover $(A \cap H) \cap(A \cap G) \subset H \cap G=\emptyset$. Suppose that $A \cap H$ and $A \cap G$ are nonempty. Then $\mathrm{A}$ is not a $\mathrm{Cl}-C l^{\star}$-connected, a contradiction. Thus, either $A \cap H=\emptyset$ or $A \cap G=\emptyset$. This implies that $A \subset H$ or $A \subset G$.

Theorem 5. If $A$ is a $C l^{\star}$-connected subset of $(X, \tau, I)$ and $A \subset B \subset A^{\star}$, then $B$ is also a $C l^{\star}$-connected subset of $X$.

Proof. Suppose that $B$ is not a $C l^{\star}$-connected subset of $(X, \tau, I)$ then there exist $C l^{\star}$-separated sets $H$ and $G$ such that $B=H \cup G$. By Theorem 3, either $A \subset H$ or $A \subset G$. Suppose that $A \subset H$. Then $A^{\star} \subset H^{\star}$. This implies that $G \subset B \subset A^{\star}$ and $G=A^{\star} \cap G \subset H^{\star} \cap G=\emptyset$, a contradiction.

Theorem 6. If $A$ is a $C l-C l^{\star}$-connected subset of $(X, \tau, I)$ and $A \subset B \subset$ $A^{\star}$, then $B$ is also a $C l-C l^{\star}$-connected subset of $X$.

Proof. Suppose that $B$ is not a $C l-C l^{\star}$-connected subset of $(X, \tau, I)$ then there exist $C l-C l^{\star}$-separated sets $H$ and $G$ such that $B=H \cup G$. By Theorem 4, either $A \subset H$ or $A \subset G$. Suppose that $A \subset H$. Then $A^{\star} \subset H^{\star}$. This implies that $G \subset B \subset A^{\star}$ and $G \subseteq A^{\star} \cap C l(G) \subset H^{\star} \cap C l(G) \subset$ $C l^{\star}(H) \cap C l(G)=\emptyset$, a contradiction.

Corollary 1. (a) If $A$ is a $C l^{\star}$-connected set in an ideal space $(X, \tau, I)$, then $A^{\star}$ is $C l^{\star}$-connected.

(b) If $A$ is a $C l-C l^{\star}$-connected set in an ideal space $(X, \tau, I)$, then $A^{\star}$ is $C l-C l^{\star}$-connected.

Corollary 2. (a) If $I \cap \tau=\emptyset$ in $(X, \tau, I)$, then for any nonempty open, $C l^{\star}$-connected set $V, C l(V)$ is also $C l^{\star}$-connected.

(b) If $I \cap \tau=\emptyset$ in $(X, \tau, I)$, then for any nonempty open, $C l-C l^{\star}-$ connected set $\mathrm{V}, \mathrm{Cl}(\mathrm{V})$ is also $\mathrm{Cl}-\mathrm{Cl}^{\star}$-connected.

Proof. (a) Follows from the Note 3.2 [7].

(b) Follows from the Note 3.2 [7].

Corollary 3. (a) If $I \cap \tau=\emptyset$ and $I \sim \tau$ in $(X, \tau, I)$, then for any nonempty open, $C l^{\star}$-connected set $G, C l(G)$ and $C l^{\star}(G)$ is also $C l^{\star}$-connected.

(b) If $I \cap \tau=\emptyset$ and $I \sim \tau$ in $(X, \tau, I)$, then for any nonempty open, $C l-C l^{\star}$-connected set $G, C l(G)$ and $C l^{\star}(G)$ is also $C l-C l^{\star}$ connected.

Proof. (a) Follows from the Note 3.2 [7]. 
(b) Follows from the Note $3.2[7]$.

Theorem 7. If $\left\{M_{i}: i \in I\right\}$ is a nonempty family of $C l^{\star}$-connected sets of an ideal space $(X, \tau, I)$ with $\bigcap_{i \in I} M_{i} \neq \emptyset$, then $\bigcup_{i \in I} M_{i}$ is $C l^{\star}$-connected.

Proof. Suppose $\bigcup_{i \in I} M_{i}$ is not $C l^{\star}$-connected. Then we have $\bigcup_{i \in I} M_{i}=$ $H \cup G$, where $H$ and $G$ are $C l^{\star}$-separated sets in $X$. Since $\bigcap_{i \in I} M_{i} \neq \emptyset$, we have a point $x \in \bigcap_{i \in I} M_{i}$. Then either $x \in H$ or $x \in G$. Suppose that $x \in H$. By Theorem 3, $M_{i} \subset H$ for all $i \in I$ and hence $\bigcup_{i \in I} M_{i} \subset H$, a contradiction.

Theorem 8. If $\left\{M_{i}: i \in I\right\}$ is a nonempty family of $C l-C l^{\star}$-connected sets of an ideal space $(X, \tau, I)$ with $\bigcap_{i \in I} M_{i} \neq \emptyset$, then $\bigcup_{i \in I} M_{i}$ is $C l-C l^{\star}$ connected.

Proof. The proof is similar with the previous Theorem.

Corollary 4. (1) If $A$ is a $C l^{\star}$-connected subset of the ideal space $(X, \tau, I)$ and $A \cap A^{\star} \neq \emptyset$, then $C l^{\star}(A)$ is a $C l^{\star}$-connected set.

(2) If $A$ is a $C l-C l^{\star}$-connected subset of the ideal space $(X, \tau, I)$ and $A \cap A^{\star} \neq \emptyset$, then $C l^{\star}(A)$ is a $C l-C l^{\star}$-connected set.

Theorem 9. Let $(X, \tau, I)$ be an ideal space, $\left\{A_{\alpha}: \alpha \in \Delta\right\}$ be a family of $C l^{\star}$-connected subsets of $X$ and $A$ be a $C l^{\star}$-connected subset of $X$. If $A \cap A_{\alpha} \neq \emptyset$, for every $\alpha$, then $A \cup\left(\cup A_{\alpha}\right)$ is $C l^{\star}$-connected.

Proof. Since $A \cap A_{\alpha} \neq \emptyset$, for each $\alpha \in \Delta$, by Theorem 7, $A \cup A_{\alpha}$ is $C l^{\star}$ connected for each $\alpha \in \Delta$. Since $\cap\left(A \cup A_{\alpha}\right) \supset A \neq \emptyset$, by Theorem 7, $A \cup\left(\cup A_{\alpha}\right)$ is $C l^{\star}$-connected.

Theorem 10. Let $(X, \tau, I)$ be an ideal space, $\left\{A_{\alpha}: \alpha \in \Delta\right\}$ be a family of $\mathrm{Cl}-C l^{\star}$-connected subsets of $X$ and $\mathrm{A}$ be a $\mathrm{Cl}-\mathrm{Cl}^{\star}$-connected subset of $X$. If $A \cap A_{\alpha} \neq \emptyset$, for every $\alpha$, then $A \cup\left(\cup A_{\alpha}\right)$ is $C l-C l^{\star}$-connected.

Proof. The proof is similar with the previous Theorem.

Recall that a subset $A$ of $(X, \tau, I)$ is called $\star$-dense-in-itself ([3])if $A \subset A^{\star}$.

Theorem 11. Let $(X, \tau, I)$ be an ideal space. If $A$ and $B$ are $C l^{\star}$-separated and $\star$-dense-in-itself subsets of $X$, and $A \cup B \in \tau$, then $A$ and $B$ are open and hence $\star$-open.

Proof. Since $A$ and $B$ are $C l^{\star}$-separated in $X$, then $A=(A \cup B) \cap\left(X \backslash B^{\star}\right)$. Since $A \cup B \in \tau$ and $B^{\star}$ is closed in $X, A$ is open in $X$.

Theorem 12. Let $(X, \tau, I)$ be an ideal space. If $A$ and $B$ are $C l-C l^{\star}$ separated subsets of $X$, and $A \cup B \in \tau$, then $A$ and $B$ are open and hence $\star$-open.

Proof. Since $A$ and $B$ are $C l-C l^{\star}$-separated in $X$, then $A=(A \cup B) \cap(X)$ $C l(B))$. Since $A \cup B \in \tau$ and $C l(B)$ is closed in $X, A$ is open in $X$. 
Definition 7. Let $X$ be an ideal space and $x \in X$. The union of all $C l^{\star}$ connected (resp. $\mathrm{Cl}-C l^{\star}$-connected) subsets of $\mathrm{X}$ containing $x$ is called the $C l^{\star}$-component (resp. $C l-C l^{\star}$-component) of $X$ containing $x$.

Theorem 13. Each $C l^{\star}$-component of an ideal space $(X, \tau, I)$ is a maximal $C l^{\star}$-connected set of $X$.

Proof. The proof follows directly from the Definition 7 .

Theorem 14. Each $C l-C l^{\star}$-component of an ideal space $(X, \tau, I)$ is a maximal $C l-C l^{\star}$-connected set of $X$.

Proof. The proof follows directly from the Definition 7 .

Theorem 15. The set of all distinct $C l^{\star}$-components of an ideal space $(X, \tau, I)$ forms a partition of $X$.

Proof. Let $A$ and $B$ be two distinct $C l^{\star}$-components of $X$. Suppose $A$ and $B$ intersect. Then, by Theorem $7, A \cup B$ is $C l^{\star}$-connected in $X$. Since $A \subset A \cup B$, then $A$ is not maximal. Thus, $A$ and $B$ are disjoint.

Theorem 16. The set of all distinct $C l-C l^{\star}$-components of an ideal space $(X, \tau, I)$ forms a partition of $X$.

Proof. The proof is similar with the previous Theorem and follows directly from the Definition 7.

Theorem 17. Let $(X, \tau, I)$ be an ideal space. Then each $C l^{\star}$-connected subset of $X$ which is both open and $\star$-closed is $C l^{\star}$-component of $X$.

Proof. Let $A$ be a $C l^{\star}$-connected subset of $X$ such that $A$ is both open and $\star$-closed. Let $x \in A$ and $C$ be the $C l^{\star}$-component containing $x$. Then $A \subset C$. Let $A$ be a proper subset of $C$. Since $A$ is open and $\star$-closed, $A \cap C l^{\star}(X \backslash A)=A \cap(X \backslash A)=\emptyset=A \cap(X \backslash A)=C l^{\star}(A) \cap(X \backslash A)$. This implies that $A \cap C l^{\star}((X \backslash A) \cap C)=\emptyset=C l^{\star}(A) \cap((X \backslash A) \cap C)$. This shows that $(A \cap C)$ and $((X \backslash A) \cap C)$ are $C l^{\star}$-separated sets, a contradiction.

Theorem 18. Let $(X, \tau, I)$ be an ideal space where $I$ is codense. Then each $C l-C l^{\star}$-connected subset of $X$ which is both open and $\star$-closed is $C l-C l^{\star}$-component of $X$.

Proof. Let $A$ be a $C l-C l^{\star}$-connected subset of $X$ such that $A$ is both open and $\star$-closed. Let $x \in A$ and $C$ be the $C l-C l^{\star}$-component containing $x$. Then $A \subset C$. Let $A$ be a proper subset of $C$. Since $A$ is open and $\star$-closed, $A \cap C l(X \backslash A)=A \cap(X \backslash A)=\emptyset=A \cap(X \backslash A)=C l^{\star}(A) \cap(X \backslash A)$. This implies that $C l(A) \cap C l^{\star}(X \backslash A)=\emptyset=C l^{\star}(A) \cap C l(X \backslash A)$ since $I$ is codense. This shows that $(A \cap C)$ and $((X \backslash A) \cap C)$ are $C l-C l^{\star}$-separated sets, a contradiction. 


\section{Connectedness And Mappings}

It is well known that $f:(X, \tau) \rightarrow(Y, \mu)$ is continuous if the inverse image of open set in $Y$ under $f$ is open in $X$ and contra-continuous ([13]) if the inverse image of open set in $Y$ under $f$ is closed in $X$

Definition 8. A function $f:(X, \tau, I) \rightarrow(Y, \mu, J)$ is said to be

(1) continuous if the inverse image of each open set in $Y$ under $f$ is open in $X$.

(2) contra-continuous if the inverse image of each open set in $Y$ under $f$ is closed in $X$.

(3) $\tau-\mu^{\star}$-continuous if the inverse image of each $\star$-open set in $Y$ under $f$ is open in $X$.

(4) $\tau^{\star}-\mu^{\star}$-continuous if the inverse image of each $\star$-open set in $Y$ under $f$ is $\star$-open in $X$.

(5) $\tau-\mu^{\star}$-contra-continuous if the inverse image of each $\star$-open set in $Y$ under $f$ is closed in $X$.

(6) $\tau^{\star}-\mu^{\star}$-contra-continuous if the inverse image of each $\star$-open set in $Y$ under $f$ is $\star$-closed in $X$.

Theorem 19. Let $f:(X, \tau, I) \rightarrow(Y, \mu, J)$ be a continuous surjection. If $X$ is $C l^{\star}$-connected, then $Y$ is connected.

Proof. Suppose that $Y$ is not connected. Then there is a nonempty clopen proper subset $A$ of $Y$. Then $f^{-1}(A)$ is nonempty clopen proper set in $X$ and hence $f^{-1}(A)$ and $X \backslash f^{-1}(A)$ constitute a $C l^{\star}$-separation of $X$, a contradiction.

Theorem 20. Let $f:(X, \tau, I) \rightarrow(Y, \mu, J)$ be a continuous surjection. If $\mathrm{X}$ is $\mathrm{Cl}-\mathrm{Cl} l^{\star}$-connected, then $\mathrm{Y}$ is $\mathrm{Cl}-\mathrm{Cl} \mathrm{l}^{\star}$-connected.

Proof. Suppose that $Y$ is not $C l-C l^{\star}$-connected. So there are disjoint nonempty sets $A$ and $B$ with $C l(A) \cap C l^{\star}(B)=C l^{\star}(A) \cap C l(B)=\emptyset$ and $Y=A \cup B$.Then $X=f^{-1}(A) \cup f^{-1}(B), f^{-1}(A) \cap f^{-1}(B)=\emptyset$ and $A$ and $B$ are open in $Y$. Since $f$ is continuous surjection, $f^{-1}(A)$ and $f^{-1}(B)$ are disjoint nonempty clopen sets in $X$ and hence $f^{-1}(A)$ and $f^{-1}(B)$ constitute a $C l-C l^{\star}$-separation of $X$, a contradiction.

Theorem 21. Let $f:(X, \tau, I) \rightarrow(Y, \mu, J)$ be a contra-continuous surjection. If $X$ is $C l^{\star}$-connected, then $Y$ is connected.

Proof. Suppose that $Y$ is not connected. So there are disjoint nonempty open sets $A$ and $B$ such that $Y=A \cup B$.Then $X=f^{-1}(A) \cup f^{-1}(B)$ and $f^{-1}(A) \cap f^{-1}(B)=\emptyset$. Since $f$ is contra-continuous surjection, $f^{-1}(A)$ and $f^{-1}(B)$ are disjoint nonempty clopen and hence $f^{-1}(A)$ and $f^{-1}(B)$ constitute a $C l^{\star}$-separation of $X$, a contradiction.

Theorem 22. Let $f:(X, \tau, I) \rightarrow(Y, \mu, J)$ be a contra-continuous surjection. If $\mathrm{X}$ is $\mathrm{Cl}-\mathrm{Cl} l^{\star}$-connected, then $\mathrm{Y}$ is $\mathrm{Cl}-\mathrm{Cl} l^{\star}$-connected.

Proof. Suppose that $Y$ is not $C l-C l^{\star}$-connected. So there are disjoint nonempty sets $A$ and $B$ with $C l(A) \cap C l^{\star}(B)=C l^{\star}(A) \cap C l(B)=\emptyset$ and 
$Y=A \cup B$.Then $X=f^{-1}(A) \cup f^{-1}(B), f^{-1}(A) \cap f^{-1}(B)=\emptyset$ and $A$ and $B$ are open sets in $Y$. Since $f$ is contra-continuous surjection, $f^{-1}(A)$ and $f^{-1}(B)$ are disjoint nonempty closed sets in $X$ and hence $f^{-1}(A)$ and $f^{-1}(B)$ constitute a $C l-C l^{\star}$-separation of $X$, a contradiction.

Theorem 23. Let $f:(X, \tau, I) \rightarrow(Y, \mu, J)$ be $a \tau^{\star}-\mu^{\star}$-continuous surjection. If $X$ is $C l^{\star}$-connected, then $Y$ is $C l^{\star}$-connected.

Proof. Suppose that $Y$ is not $C l^{\star}$-connected. So there are nonempty disjoint sets $A$ and $B$ with $A \cap C l^{\star}(B)=C l^{\star}(A) \cap B=\emptyset$ and $Y=A \cup B$. Then $A$ and $B$ are $\star$-open in $Y$ so that $f^{-1}(A)$ and $f^{-1}(B)$ are $\star$-open and $\star$-closed in $X$. The sets $f^{-1}(A)$ and $f^{-1}(B)$ constitute a $C l^{\star}$-separation of $X$, a contradiction.

Theorem 24. Let $f:(X, \tau, I) \rightarrow(Y, \mu, J)$ be a $\tau^{\star}-\mu^{\star}$-contra-continuous surjection. If $X$ is $C l^{\star}$-connected, then $Y$ is $C l^{\star}$-connected.

Proof. Suppose that $Y$ is not $C l^{\star}$-connected. So there are nonempty disjoint sets $A$ and $B$ with $A \cap C l^{\star}(B)=C l^{\star}(A) \cap B=\emptyset$ and $Y=A \cup B$. Then $A$ and $B$ are $\star$-open in $Y$ so that $f^{-1}(A)$ and $f^{-1}(B)$ are also $\star$-closed in $X$. Since $X=f^{-1}(A) \cup f^{-1}(B)$ and $f^{-1}(A) \cap f^{-1}(B)=\emptyset$. The sets $f^{-1}(A)$ and $f^{-1}(B)$ constitute a $C l^{\star}$-separation of $X$, a contradiction.

Theorem 25. Let $f:(X, \tau, I) \rightarrow(Y, \mu, J)$ be a $\tau-\mu^{\star}$-continuous function.Then $f$ is $\tau^{\star}-\mu^{\star}$-continuous and continuous function.

Proof. Follows from the fact that $\tau \subseteq \tau^{\star}$ and $\mu \subseteq \mu^{\star}$

Corollary 5. Let $f:(X, \tau, I) \rightarrow(Y, \mu, J)$ be a $\tau-\mu^{\star}$-continuous surjection. If $X$ is $C l^{\star}$-connected, then $Y$ is $C l^{\star}$-connected.

Corollary 6. Let $f:(X, \tau, I) \rightarrow(Y, \mu, J)$ be a $\tau-\mu^{\star}$-continuous surjection. If $\mathrm{X}$ is $\mathrm{Cl}-\mathrm{Cl} l^{\star}$-connected, then $\mathrm{Y}$ is $\mathrm{Cl}-\mathrm{Cl}^{\star}$-connected.

Theorem 26. Let $f:(X, \tau, I) \rightarrow(Y, \mu, J)$ be a $\tau-\mu^{\star}$-contra-continuous function. Then $f$ is $\tau^{\star}-\mu^{\star}$-contra-continuous and contra-continuous function.

Proof. Follows from the fact that $\tau \subseteq \tau^{\star}$ and $\mu \subseteq \mu^{\star}$.

Corollary 7. Let $f:(X, \tau, I) \rightarrow(Y, \mu, J)$ be a $\tau-\mu^{\star}$-contra-continuous surjection. If $X$ is $C l^{\star}$-connected, then $Y$ is $C l^{\star}$-connected.

Corollary 8. Let $f:(X, \tau, I) \rightarrow(Y, \mu, J)$ be a $\tau-\mu^{\star}$-contra-continuous surjection. If $\mathrm{X}$ is $\mathrm{Cl}-\mathrm{Cl}^{\star}$-connected, then $\mathrm{Y}$ is $\mathrm{Cl}-\mathrm{Cl}^{\star}$-connected.

Definition 9. A function $f:(X, \tau, I) \rightarrow(Y, \mu, J)$ is said to be

(1) $\tau^{\star}-\mu^{\star}$-homeomorphism if $f:\left(X, \tau^{\star}(I)\right) \rightarrow\left(Y, \mu^{\star}(J)\right)$ is a homeomorphism.

(2) Ideal homeomorphism if it is both a homeomorphism and $\tau^{\star}-\mu^{\star}-$ homeomorphism

Theorem 27. Every $\tau^{\star}-\mu^{\star}$-homeomorphism is $\tau^{\star}-\mu^{\star}$-continuous.

Proof. The proof follows directly from the Definition 9. 
Corollary 9. A $\tau^{\star}-\mu^{\star}$-homeomorphism preserves $C l^{\star}$-connectedness.

Corollary 10. A homeomorphism preserves $C l-C l^{\star}$-connectedness.

Corollary 11. An Ideal homeomorphism preserves $C l^{\star}$-connectedness and $\mathrm{Cl}-\mathrm{Cl} l^{\star}$-connectedness.

\section{REFERENCES}

[1] J. Dontchev, M. Ganster, D. Rose Ideal resolvability, Topology Appl. 93 (1999) 1-6.

[2] E. Ekici, T. Noiri, Connectedness in ideal topological spaces, Novi Sad J. Math. 38 (2008) 65-70.

[3] E. Hayashi, Topologies defined by local properties, Math. Ann. 156 (1964) 205-215.

[4] D. Jankovic, T. R. Hamlett, New topologies from old via ideals, Amer. Math. Monthly 97 (1990) 295-310.

[5] D. Jankovic, T. R. Hamlett, Compatible extensions of ideals, Boll. Un. Mat. Ital. 7 (1992) 453-465.

[6] K. Kuratowski, Topology, Vol. I. New York, Academic Press, 1966.

[7] S. Modak, C. Bandyopadhyay, «-topology and generalized open sets, Soochow J. Math. 32 (2006) 201-210.

[8] S. Modak, C. Bandyopadhyaya, Ideals and generalized open sets, News Bull. Cal. Math. Soc. 29 (2006) 9-12.

[9] N. Sathiyasundari, V. Renukadevi, Note on $\star$-connected ideal spaces, Novi Sad J. Math. 42 (2012) 15-20.

[10] V. Vaidyanathswamy, The locaization theory in set topology, Proc. India Acad. Sci. 20 (1945) 51-61.

[11] S. Modak, T. Noiri, Connectedness of ideal topological spaces, Filomat 29 (2015) 661-665

[12] E. Ekici, T. Noiri, ^-Hyperconnected ideal topological spaces, Analele Stiintifile Ale Universitatii "AL.I. CUZA" Din Iasi(S.N.) Matematica, Tomul LVIII, 2012, f.1 DOI:10.247/v10157-011-0045-9.

[13] J. Dontchev, Contra-continuous functions and strongly s-closed spaces, Internat. J. Math. Math. Sci. 19 (1996) 303-310.

Department of Mathematics, Atmaram Sanatan Dharma College, University of Delhi, New Delhi-110021, India

Email address: brijkishore.tyagi@gmail.com

Department of Mathematics,

University of Delhi, New Delhi - 110007, India

Email address: manojmnj27@gmail.com

Department of Mathematics,

University of Delhi, New Delhi-110007, India

Email address: sumitkumar405@gmail.com 